\title{
REFLEXIONES SOBRE LA ENSEÑANZA DEL “OFICIO" DE HISTORIADOR A PARTIR DE LA PRÁCTICA DOCENTE EN MISIONES, ARGENTINA
}

\author{
María Cecilia Gallero \\ Consejo Nacional de Investigaciones Científicas y Técnicas (Argentina) \\ mariaceciliagallero@gmail.com
}

\begin{abstract}
RESUMEN: Este artículo analiza la enseñanza de la escritura de la historia a partir de mi experiencia docente en el dictado del "Seminario de Metodología de la Investigación Histórica" a los alumnos del último año del Profesorado de Historia del Instituto Antonio Ruiz de Montoya en Posadas, Misiones, Argentina. Una provincia que desde su situación histórica-geográfica se encuentra en los márgenes nacionales, los que a su vez están agudizados por su propia condición de frontera con los países de Brasil y Paraguay, y por el vacío marginal de los estudios históricos realizados desde los centros académicos.

Una de las principales competencias en enseñar cómo escribir una monografía fue afianzar ciertas destrezas que se plasman o evidencian en la "teoría histórica". Los alumnos, futuros profesores, aunque se dedicaran luego a la enseñanza de la historia, debían saber aplicar los fundamentos metodológicos y lo que implica la investigación histórica.
\end{abstract}

Palabras clave: investigación histórica, metodología, campos de estudio, práctica docente, regiones de frontera.

\section{REFLECTIONS ON THE TEACHING OF HISTORIAN'S "PROFESSION" THROUGH THE PRACTICE IN THE TRAINING OF FUTURE TEACHER IN MISIONES, ARGENTINA}

ABSTRACT: This article analyses the teaching of history writing based on my own experience in the "Seminar on the Methodology of Historical Research" 
to the final course students of the History Bachelors of the Antonio Ruiz de Montoya Institute in Posadas, Misiones, Argentina. This province is placed on the national margins, situation that is intensified by its own border with Brazil and Paraguay, and by the marginal lack of historical studies carried out by academic centers.

One of the main competences to teach was how to write a monography to strengthen certain skills that are embodied or evidenced in "historical theory". The students, future teachers, although they would later dedicate themselves to the teaching of history, had to know how to apply the methodological foundations and what historical research entails.

Keywords: Historical research, methodology, fields of study, teaching practice, frontier regions.

Recibido: 14 de enero de 2021 Aceptado: 27 de abril de 2021

"La historia [history] aparece cada vez que ocurre un acontecimiento lo suficientemente importante para iluminar su pasado. Entonces la masa caótica de sucesos pasados emerge como un relato [story] que puede ser contado, porque tiene un comienzo y un final"1.

\section{Introducción}

La historia, ¿es una disciplina teórico-práctica? ¿puede evidenciarse la teoría en la escritura de la historia como narración de hechos o sucesos? ¿qué teoría?

Por lo general, los historiadores tendemos a dar prioridad a los hechos, y muchas veces damos por sentada la teoría. En los últimos tiempos se ha hecho hincapié en la diferencia entre "la narración histórica de los hechos" y la "verdad de los acontecimientos sucedidos". Sin embargo, la formación en una carrera "histórica" debería brindar las herramientas para salvar los inconvenientes de un relato episódico o netamente descriptivo. Entonces, ¿qué herramientas dar a los alumnos que aspiran a ser profesores de la materia? ¿Cómo hacer para que puedan percatarse de que su relato [story] aparece como historia [history] a partir de la investigación que realizan?

1. Hannah ARENDT: De la historia a la acción, Barcelona, Paidós, [1953] 1998, p. 41. Agradezco a la profesora Karina Dohmann su acompañamiento y apoyatura en las mesas de exámenes finales de la materia, así como su lectura y observaciones realizadas a este artículo. En este último punto también doy las gracias a mi profesora de "Introducción a la historia", Susana Frías, y a mi colega Luz Pyke. 
Durante casi diez años dicté el "Seminario de Metodología de la Investigación Histórica" ${ }^{2}$, como cierre de esta etapa vuelco en este artículo los resultados y reflexiones de mi experiencia docente con los alumnos de cuarto año del Profesorado de Historia del Instituto Antonio Ruiz de Montoya en Posadas, Misiones, Argentina.

La enseñanza de la escritura de la historia me demandó un triple desafío, por un lado, dar a entender los requisitos mínimos de una monografía, por otro, pulir la destreza literaria, y finalmente, plantear la importancia de la teoría ${ }^{3}$. Aunque hablar de teoría parezca bastante abstracto, la misma implica algo tan simple como conceptualizar el objeto de estudio, elegir o construir una periodización adecuada y evitar problemas comunes, como la enumeración de sucesos en un escrito cronológico, la transcripción de documentos o entrevistas sin contextualización o problematización, y especialmente, la falta de explicitación del marco teórico-metodológico.

Es decir, una de las principales competencias al enseñar cómo escribir una monografía fue afianzar ciertas destrezas que se plasman o evidencian en la "teoría histórica". Para mi era importante que, aún dedicándose luego a la enseñanza de la historia, tuvieran una visión clara de lo que significa investigar, especialmente en el campo de la historia. ¿Cómo transmitir el "oficio" del historiador ${ }^{4}$ ?

"La práctica hace al maestro" dice un conocido dicho popular, y fue en la práctica dónde se hicieron evidentes los problemas, y fue en la resolución de los mismos que fuimos encontrando soluciones de manera conjunta. Por lo tanto, mi interés es desarrollar e iluminar este punto: en primer lugar, realizaré un breve contexto sobre la investigación histórica en Misiones; en segundo lugar, abordaré a partir de la práctica el análisis de la producción de los trabajos monográficos presentados a examen final; en tercer lugar, intentaré enmarcar los pasos teórico-metodológicos que fui enseñando; para finalmente, cerrar con algunas conclusiones sobre la práctica docente en la investigación histórica.

2. En el año 2016, hubo un cambio en los planes de estudio a partir del cual la materia pasó a denominarse "Metodología de la Investigación Histórica" en Cuarto Año del Profesorado de Historia, con una reducción horaria (de cuatro a tres horas reloj).

3. En esta teoría se dejaron de lado aspectos de la Filosofía de la Historia o de Historia conceptual, pues aunque estaban implícitas en mi enseñanza, me resultó imposible aplicarlas en la práctica.

4. Resalto la palabra "oficio" porque aunque haya sido utilizada para marcar la profesión del historiador (Jacques LE GOFF (dir.): Pensar la Historia, Barcelona, Paidós, 2005, p. 104 y Enrique MORADIELLOS: El oficio de historiador, Madrid, Siglo XXI, 2005), quiero remarcar que "oficio" implica un dominio disciplinar y un conocimiento profundo de la propia actividad laboral, y como define una de las acepciones de la Real Academia Española, que "sabe muy bien lo que se lleva entre manos, tiene mucho oficio". 


\section{Misiones: haciendo historia desde los márgenes}

La provincia de Misiones ${ }^{5}$ lleva su nombre como herencia de las "Misiones Jesuíticas" ${ }^{\prime \prime}$, un escenario que marcó un proceso que sólo puede ser visualizado en el marco de la historia de larga duración ${ }^{7}$ y que delimitó de algún modo una "región histórica" ${ }^{8}$, una región que es visualizada como un "margen" de la nación Argentina, un margen que a su vez está agudizado por su propia condición de frontera con los países de Brasil y Paraguay. La historiadora Ángela Perié de Schiavoni sostiene que existe una "región histórica misionera", pues la actual provincia de Misiones, constituye el núcleo de un área en donde se desarrolló la experiencia socio-económica cultural de las "Misiones".

5. Misiones es el tercer distrito más pequeño del país, después de Tucumán y la Ciudad Autónoma de Buenos Aires: sus 29.801 kilómetros cuadrados $\left(\mathrm{km}^{2}\right)$ representan el 0,8\% del total de la superficie nacional. En rigor, se trata de una jurisdicción argentina ubicada en medio de los países contiguos de Brasil y Paraguay, y apenas un $20 \%$ de su territorio, aproximadamente, limita con la provincia argentina de Corrientes. La capital provincial es Posadas, su población es de 324.756 habitantes en un total provincial de 1.101.593, según el censo de 2010. Gobierno de Misiones, Gran Atlas de Misiones. Posadas, 2012.

6. "Misiones Jesuíticas" se conoce a la experiencia evangelizadora de la Compañía de Jesús -a inicios del siglo XVII- que delimitó las fronteras de un territorio, que mantuvo características semejantes una vez consolidado. En lo que hoy es la República Argentina se localizaban los pueblos de Corpus, San Ignacio Miní, Loreto, Santa Ana, Candelaria, San José, Apóstoles, Concepción, Mártires, Santa María la Mayor, San Javier (en la actual Provincia de Misiones), San Carlos, Santo Tomé, La Cruz y Yapeyú (en la actual Provincia de Corrientes). En Paraguay estaban las reducciones de Itapúa, Trinidad, Jesús, San Ignacio Guazú, Nuestra Señora de la Fe, Santa Rosa, San Cosme y Damián y Santiago. Mientras que en Brasil se ubicaban San Miguel, San Nicolás, San Luis, San Lorenzo, San Juan, Santo Ángel y San Borja. AI concluir el primer tercio del siglo XVIII los treinta pueblos guaraníes de las Misiones Jesuíticas habían alcanzado el cenit de su población: 141.000 habitantes en 1732, con un promedio de 4500 almas por pueblo. Sus estancias ocupaban extensos campos de Río Grande y de la Banda Oriental y su producción satisfacía plenamente las necesidades de aquellas comunidades. Para ampliar el tema véase: Guillermo FURLONG: Misiones y sus Pueblos Guaraníes, Buenos Aires, Imprenta Balmes, 1962; Antonio ASTRAIN: Jesuitas, Guaraníes y Encomenderos: historia de la Compañía de Jesús en el Paraguay, Asunción, CEPAG, 1995; y Ernesto MAEDER: Aproximación a las misiones guaraníticas, Buenos Aires, EDUCA, 1996.

7. Fernand Braudel realiza una distinción entre el tiempo corto y la larga duración, y para ello se apoya en la noción de estructura como una herramienta analítica, en la cual la larga duración es como "un ensamblaje, una arquitectura; pero más aún, una realidad que el tiempo tarda enormemente en desgastar y en transportar". Fernand BRAUDEL: La Historia y las Ciencias Sociales, Madrid, Alianza, 1970, p. 70.

8. La "Región Histórica" "constituye una totalidad relativa, socioeconómica y política sometida a procesos de estructuración /desestructuración/ reestructuración, que condicionan: 1. la variabilidad temporal de sus límites; 2 . las cambiantes relaciones intra e interregionales; 3. la organización del soporte espacial." Fernando JAUME, Carlos GONZALES VILLAR, Yolanda URQUIZA y Lila SINTES: Notas sobre la Historia de Misiones. El proceso de Constitución de la Región Histórica, Documentos de Trabajo, Proyecto POBUR, UNaM-CONICET, [s.f.], p. 6.

9. Ángela PERIÉ DE SCHIAVONI: "El estudio de la colonización: sus fuentes documentales", Estudios Regionales, Año 11, 2 (mayo 2002), p. 10. 
Sin embargo, aunque exista este largo bagaje histórico, el historiador está muy lejos de disponer de repositorios que faciliten los trabajos heurísticos en esta provincia ${ }^{10}$. Al considerar la documentación histórica deben diferenciarse dos períodos. Uno anterior a la creación del Territorio Nacional de Misiones (1881), etapa de la cual no se conservan documentos en archivos públicos de la provincia, pero sí en otros fuera de ella. La diáspora postjesuítica (1767), los conflictos y movimientos de población que le sucedieron y la inestabilidad institucional, provocaron que quien estudie este período en la actualidad deba buscar información en los siguientes repositorios: Archivo General de la Nación (Buenos Aires) y Archivo General de la Provincia de Corrientes en Argentina; Archivo Nacional de Asunción en Paraguay; Archivo General de Indias (Sevilla) en España; Biblioteca Nacional de Río de Janeiro en Brasil; Archivo General de la Nación (Montevideo) en Uruguay; entre otros. El siguiente período, posterior a la territorialización de Misiones, cuenta con algún grado de conservación y sistematización de documentación, particularmente la producida por las instituciones estatales. En este período, además deben distinguirse los documentos producidos mientras era Territorio Nacional, en gran parte en el Archivo General de la Nación en la sección del Ministerio del Interior, y en parte en el propio Archivo General de la Gobernación de Misiones -en Posadas-, ubicado en el mismo edificio en el cual se desarrolla la administración institucional (1881-1953 como Territorio Nacional, 1953 al presente como provincia).

Al vacío de información sistematizada y a la falta de instituciones capaces de convertirse en referentes de consulta, se suma un vacío institucional de larga data en relación a las cuestiones históricas y culturales ${ }^{11}$. Se puede mencionar el impulso Ilevado a cabo por la Junta de Estudios Históricos de Misiones, que había sido creada en 1939 y fue reorganizada nuevamente en 1983.

En este vacío "marginal" respecto a los estudios históricos realizados desde los centros académicos, como Buenos Aires o Córdoba ${ }^{12}$, hay que hacer una distinción en los perfiles institucionales dedicados a la formación de docentes de historia en Misiones. En la década de 1960 surgen casi paralelamente las dos instituciones más importantes que se dedican a su formación. Por un lado, el

10. Yolanda URQUIZA: "Tras las Huellas de la Ciudadanía en un Territorio de Frontera", Cuadernos del Sur-Serie Historia, 30-31 (2002), p. 160. Véase también: Ernesto MAEDER: "Los Archivos...", pp. 177-183; María Angélica AMABLE: La documentación histórica en los Archivos Provinciales, Posadas, Tesis de Grado Inédita, Misiones, Universidad Nacional de Misiones, 1996; y, Ángela PERIÉ DE SCHIAVONI: "El estudio de la colonización...", pp. 7-11.

11. Héctor JAQUET: Haciendo Historia en la Aldea, Misiones, 1996, Misiones, s.e., 2002.

12. Buenos Aires y Córdoba son dos ciudades clave en la organización de la nación, ambas albergaron desde 1821 y 1613 respectivamente los centros académicos universitarios más antiguos del país. 
"Instituto Superior Antonio Ruiz de Montoya"13 surgió en 1960 a instancias del Monseñor Jorge Kemerer luego de que se aprobara la habilitación de universidades privadas para emitir títulos profesionales durante la presidencia de Arturo Frondizi (1958-1962). Los objetivos principales eran "ofrecer a la juventud misionera la posibilidad de cursar estudios superiores" y "cubrir la necesidad en cuanto a docencia especializada" en la provincia. Fueron cuatro las carreras fundacionales: Profesorado en Filosofía y Pedagogía, Profesorado en Castellano, Literatura y Latín, Profesorado en Geografía y Profesorado en Historia ${ }^{14}$. Por otra parte, dentro del ámbito universitario se fue consolidando la carrera en Historia con la creación del "Instituto Superior del Profesorado" por el gobierno Provincial en 1961, el cual más tarde fue incorporado a la Universidad Nacional de Misiones en $1974^{15}$. Desde sus inicios contribuyó a la formación de profesores y licenciados en Historia, siendo esta última titulación destinada especialmente a la investigación.

Ahora bien, en este breve contexto hay que tener en cuenta que las últimas leyes nacionales (Ley Federal de Educación Nº 24195 de 1993 y Ley de Educación Nacional No 26206 de 2006) distinguieron entre instituciones de gestión "estatal" y de gestión "privada", reforzando la idea de que estas últimas son "agentes" del Estado. En este sentido, tanto a nivel primario, secundario como universitario, su actividad está dotada de atributos "públicos" a partir de lineamientos definidos por la autoridad estatal (estructura y contenidos curriculares; criterios de permanencia, promoción y evaluación de los alumnos; titulación de quienes están autorizados para enseñar; validez de las credenciales emitidas; entre otras) ${ }^{16}$.

De modo que dentro de las principales diferencias entre ambas instituciones, además de ser una pública y otra de gestión privada, se encuentra la propia orientación institucional: la universidad con un perfil destinado a la investigación y el Instituto Montoya a la formación de un profesorado abocado especialmente al nivel secundario (13-17 años).

13. Página web del Instituto Superior Antonio Ruiz de Montoya: https://www.isparm.edu. ar/ [Consultado 01/12/2020].

14. María Eva LESCANO y Silvia VENANZI: 1960-2010, Instituto Superior Antonio Ruiz de Montoya, 50 Aniversario, Posadas, 2010.

15. El 20 de abril de 1974 el Instituto Superior del Profesorado fue incorporado a la Universidad Nacional de Misiones (UNaM), la cual había sido creada un año antes. Por otra parte, hay que tener en cuenta que este instituto se fusionó con la Escuela de Servicios Sociales, que dependía de la Universidad Nacional del Nordeste desde 1966, con lo cual se dio inicio a la Facultad de Humanidades y Ciencias Sociales (UNaM) el 17 de noviembre de 1980. Veáse la Historia de la Universidad Nacional de Misiones, en el link: https://www.unam.edu.ar/index.php/ institucional/historia, [Consultado 12/12/2020], y la página web de la Facultad de Humanidades y Ciencias Sociales: https://www.fhycs.unam.edu.ar/portada/ [consultado 01/12/2020].

16. Gustavo GAMALLO: "La "publificación" de las escuelas privadas en Argentina". Revista SAAP. Publicación de Ciencia Política de la Sociedad Argentina de Análisis Político, vol. 9, 1 (mayo 2015), pp. 43-74. 
Para terminar este apartado, interesa añadir, como señala Pablo Buchbinder, que en el análisis de la evolución de la disciplina en la enseñanza superior, es "indispensable para una historia de la historiografía, de la formación, las orientaciones y, consecuentemente, del perfil de los historiadores", si bien este autor, realiza "una primera aproximación al estudio de la evolución de la enseñanza superior de la historia durante la primera mitad del siglo XX en la Argentina"17, se concentra particularmente en el caso de la Facultad de Filosofía y Letras de la Universidad de Buenos Aires, con lo cual, la realidad misionera queda abierta a futuras investigaciones historiográficas.

\section{Metodología de la investigación en la práctica}

Uno de los principales objetivos de la materia es que los alumnos aprendan los fundamentos metodológicos de la investigación histórica y que puedan poner en la práctica los conocimientos teóricos adquiridos en materias como "Introducción a la Historia" e "Historiografía". Para ello, de manera individual, cada alumno debía realizar una investigación histórica cuyo producto final consistía en una monografía en la que pusieran en juego un trabajo creador mediante el manejo de fuentes, bibliografía y el empleo de una metodología científica. Mi objetivo principal era que los alumnos lograran en el "hacer" el conocimiento necesario para profundizar los saberes aprendidos y conocer diferentes métodos para apropiarse de una destreza que fortaleciera el dominio disciplinar y aprendieran el "oficio".

He comenzado cada ciclo lectivo con "Los hábitos del pensamiento riguroso"18, enumerados en seis habilidades que había que ir trabajando y reforzando a lo largo de la cursada, a saber: hábito de la definición, de la distinción, de la relación y de la causalidad, de la sistematización, de la crítica y de la síntesis. Posteriormente, trabajábamos sobre lecturas de Marrou, De Certeau, Bloch, Le Goff, Arostegui y Moradiellos ${ }^{19}$. Entre lectura y planteamiento de los principios de la investigación ${ }^{20}$, se realizaba una visita a la biblioteca del

17. Pablo BUCHBINDER: "¿Qué debe saber un historiador? Reflexiones sobre los modelos curriculares y la enseñanza superior de la historia en la Argentina durante el siglo XX", Clio \& Asociados, 15 (2011), pp. 157-177.

18. Roberto BRIE: Los hábitos del pensamiento riguroso, Buenos Aires, Ed. del Viejo Aljibe, 1998.

19. Henri MARROU: Del Conocimiento Histórico, Buenos Aires, 1985; M. DE CERTEAU: "La operación histórica", en Jacques LE GOFF (dir.): Hacer la historia, Barcelona, Laia, 1984; Marc BLOCH: Introducción a la Historia, México, FCE, 2000; Jacques LE GOFF (dir.): Pensar...; Julio AROSTEGUI: La investigación Histórica: Teoría y Método, Barcelona, Crítica, 2001; y E. MORADIELLOS: El oficio....

20. Ciro Flamarión CARDOSO: Introducción al trabajo de la investigación histórica, Barcelona, Crítica, 1989, p. 173. 
Instituto, Ilamada "Nicolás Yapuguay"21. Recorrer los anaqueles de la "Sección Misiones", revisar producciones sobre historia de poblamiento, historia política o trabajos sobre economía. Lentamente ir logrando que se interesaran por un tema. Algunos ya lo tenían definido, ya sea porque les interesaba saber más sobre un proceso que no habían logrado entender bien o porque se sentían ligados a esa historia (la historia de su pueblo, iglesia, escuela primaria o secundaria). Este primer paso que parecía sencillo debía estar sustentado sobre una temática que los inspirara pero que, simultáneamente, tuviera significación histórica. Si al principio el abanico de temas era amplio, luego se iba definiendo según las posibilidades reales. En este proceso, se iban "encauzando" los intereses a medida que se planteaban interrogantes y se delimitaba la hipótesis; lograr que la misma fuera "aceptable", era un rompecabezas colectivo que íbamos trabajando conjuntamente a medida que redactábamos los objetivos, explayábamos la metodología y tratábamos de fundamentar el tema.

El punto siguiente, aunque no menos azaroso, era realizar el estado de la cuestión. La mayoría de las veces resultaba "exiguo material", "inexistente bibliografía", "tema escasamente tratado", y en aquellos temas más conocidos, un continuo reescribir sobre la importancia de ese material y la contribución que se pensaba realizar desde una nueva mirada. En los primeros años intenté que aplicaran la cita con nota a pie de la Academia Nacional de la Historia, pero ante las dificultades técnicas en el uso del programa Word, opté por explicar en qué consistía, y simplemente bogar porque adoptaran correctamente las normas APA (autor, año y ¡número de página!).

A lo largo de casi diez años -entre septiembre de 2011 y diciembre de 2019-, dirigí poco más de doscientos trabajos monográficos. Realicé un registro anual volcado en una ficha individual de cada alumno, en la que además, de los datos personales anotaba sus intereses, el tema que desarrollaban y las dificultades presentadas. Luego esta información me servía al momento de tomar el examen final, en los que también consignaba la fecha y el resultado/nota final del escrito y del oral.

El sustrato empírico de este "fichaje" es el punto de partida para el análisis que estoy realizando en este artículo: sobre un total de 212 monografías (100\%), se analizaron aquellas que llegaron a defenderse en examen final 153 (72,7\%), dejando de lado las $58(27,3 \%)$ que no se presentaron a rendir mientras estuve a cargo de la materia, desconozco si lo hicieron con posterioridad a mi retiro.

De los trabajos monográficos analizados, los campos de estudio de la Historia fueron principalmente relativos a la historia regional y de Misiones. En el siguiente cuadro se realiza una síntesis de los mismos:

21. Sitio web de la biblioteca: https://www.isparm.edu.ar/servicios. php?pageid=bibliotecayapuguay [consultado 12/01/2021]. 
Cuadro 1. Campos históricos y temario aproximado de las monografías rendidas en Metodología de la Investigación (2011-2019).

\begin{tabular}{|c|c|c|}
\hline Campos de estudio & \begin{tabular}{|c|} 
Títulos abreviados a modo de ejemplo de los \\
temas monográficos
\end{tabular} & Porcentaje \\
\hline $\begin{array}{c}\text { Historia del pobla- } \\
\text { miento y coloniza- } \\
\text { ción de Misiones }\end{array}$ & $\begin{array}{c}\text { Historia Ituzaingó (Corrientes); Munici- } \\
\text { palización de Colonia Delicia (1978); } \\
\text { Migración paraguaya en Mado; Cartogra- } \\
\text { fía histórica; La colonización polaca de } \\
\text { Apóstoles; Los ucranianos de Posadas; } \\
\text { Brasileños en San Javier; Poblamiento de } \\
\text { Campo Viera; Poblamiento de Montecar- } \\
\text { lo; Jardín América; Andresito; Aristóbulo } \\
\text { del Valle; San Antonio y sus límites; Co- } \\
\text { lonia Delicia; Colonia Aurora Guaraní; } \\
\text { Garuhapé; Puerto Esperanza; San Vicen- } \\
\text { te; Villa Cabello; Villa Urquiza; entre } \\
\text { otros. }\end{array}$ & $27 \%$ \\
\hline Historia Económica & $\begin{array}{l}\text { El Ferrocarril; La Oleaginosa de Campo } \\
\text { Grande; La evolución de la explotación } \\
\text { yerbatera; La Actividad tabacalera en } \\
\text { Bonpland; La Empresa Arriazu, Moure y } \\
\text { Garrasino; La Cachuera; Celulosa Argen- } \\
\text { tina (1942-2008); Energía Eléctrica en } \\
\text { Cerro Azul; Yerbartera Compadre; Yerba- } \\
\text { tera del Nordeste; Yerbatera Mate Rojo; } \\
\text { Cooperativa Agrícola de Ruiz de Monto- } \\
\text { ya; Ferrobarcos en Posadas; La Estancia } \\
\text { Santa Inés; Yacyretá; La electricidad en } \\
\text { Misiones; Cooperativa de Servicios Can- } \\
\text { delaria Ltda; entre otros. }\end{array}$ & $14 \%$ \\
\hline $\begin{array}{l}\text { Historia Social y } \\
\text { cultural }\end{array}$ & $\begin{array}{c}\text { La vida Cotidiana en Posadas; Hospital } \\
\text { Ramón Madariaga; El Puente San Roque } \\
\text { González de Santa Cruz; La fiesta del } \\
\text { inmigrante; La fiesta nacional del Té; La } \\
\text { fiesta de la madera en San Vicente; Club } \\
\text { de Fútbol Guaraní Antonio Franco; Cine } \\
\text { Teatro de Oberá; La Biblioteca Popular } \\
\text { de Posadas; Biografía de Samuel Fuxman; } \\
\text { La casa de Gobierno; Las Paseras; El } \\
\text { Mensú; entre otros. }\end{array}$ & $13 \%$ \\
\hline
\end{tabular}




\begin{tabular}{|c|c|c|}
\hline Campos de estudio & \begin{tabular}{|c|} 
Títulos abreviados a modo de ejemplo de los \\
temas monográficos
\end{tabular} & Porcentaje \\
\hline $\begin{array}{l}\text { Historia de la } \\
\text { Educación }\end{array}$ & $\begin{array}{l}\text { La educación primaria en Misiones 1945- } \\
\text { 1955; Escuela Normal Mixta de Posadas: Es- } \\
\text { cuela Normal N } 2 \text { de Montecarlo; Escuela } \\
\text { № } 503 \text { de San Ignacio; La fiesta del docen- } \\
\text { te; El Bachillerato Humanista; La educación } \\
\text { en el proceso de Reorganización Nacional } \\
\text { (1976-1983); Historia de la Escuela de ofi- } \\
\text { ciales de la Policía Manuel Belgrano; Histo- } \\
\text { ria del Colegio San Basilio Magno; Escuela } \\
\text { Normal Mixta de Brasil; entre otros. } \\
\end{array}$ & $13 \%$ \\
\hline Historia Política & $\begin{array}{c}\text { El Peronismo en Misiones (1945-1955); El } \\
\text { gobernador Humada y la salud; Políticas } \\
\text { agrarias de Humada; La provincialización } \\
\text { de Misiones (1953); El Movimiento Agrario } \\
\text { Misionero; La Cámara de representantes } \\
\text { (1943-2007); El partido socialista en Misio- } \\
\text { nes, Historia de la Policía; Los gobernadores } \\
\text { democráticos (1983-2011); Las mujeres en } \\
\text { la política; El Radicalismo en Misiones; en- } \\
\text { tre otros. } \\
\end{array}$ & $12 \%$ \\
\hline $\begin{array}{l}\text { Historia del siglo } \\
\qquad \text { XIX }\end{array}$ & $\begin{array}{l}\text { Andresito desde una mirada historiográfica; } \\
\text { Misiones como parte de Corrientes (1830- } \\
\text { 1880); Las reuniones secretas de la masone- } \\
\text { ría en la Revolución de Mayo; El directorio } \\
\text { de Juan Martín de Pueyrredón, entre otros. }\end{array}$ & $12 \%$ \\
\hline $\begin{array}{l}\text { Historia de la } \\
\text { Iglesia }\end{array}$ & $\begin{array}{l}\text { La Catedral de Posadas, La Diócesis de } \\
\text { Iguazú; Biografía del Obispo Kemerer, His- } \\
\text { toria de la Iglesia de Posadas; Iglesia de la } \\
\text { Inmaculada; entre otras. }\end{array}$ & $5 \%$ \\
\hline Historia ambiental & $\begin{array}{c}\text { Movimientos ecologistas de Misiones; Mi- } \\
\text { nisterio de Ecología; El impacto de la Repre- } \\
\text { sa Urugua-í; La reserva del Cuña Pirú. }\end{array}$ & $4 \%$ \\
\hline
\end{tabular}

Una vez que el plan de trabajo se mostraba viable y era aprobado al finalizar el primer cuatrimestre (Abril-Julio), se pasaba a la segunda etapa: la investigación y escritura de la monografía, que coincidía temporalmente con el segundo cuatrimestre (Agosto-Noviembre). Si en el transcurso del año habían logrado ir trabajando el tema, la meta de rendir en las mesas de diciembre o febrero era viable, lo cual fue posible para un $25 \%$ de los alumnos. Mientras que un $42 \%$ rindieron al año siguiente, un 26\% aproximadamente en dos años, un $12 \%$ en 
el tiempo justo de vencerse la regularidad y un 3\% con prórroga excepcional. Téngase en cuenta que la regularidad en la materia se mantiene por un período de dos años y medio. Las monografías que resultaron aprobadas en el examen final con un promedio entre el escrito y el oral mayor a ocho, se encuentran archivadas en la Biblioteca Nicolás Yapuguay, las que sirven en mayor medida como punto de partida para nuevas investigaciones.

Ejercitar el pensamiento crítico y la reflexión a través de ejercicios y lecturas del campo teórico, metodológico e histórico era una labor cotidiana en cada clase. Por lo general se iniciaba con una explicación teórico-práctica, luego se dedicaba el tiempo a trabajar en lecturas propias del tema a trabajar y a la atención de consultas sobre bibliografía o documentación.

El número de alumnos de cada cohorte influía en el desempeño general: entre 15-20 alumnos se podía realizar un seguimiento más personalizado, en tanto que se dificultaba el trabajo individual con 20-30 o más alumnos; en este último caso se optaba por organizar grupos de tres a cuatro alumnos que trabajaran la misma metodología o el mismo campo histórico para la atención de consultas, recomendación de bibliografía o solución a problemas comunes.

Asimismo, la organización por campos históricos era utilizada para la distribución grupal para la visita al Archivo General de la Gobernación de Misiones $^{22}$, es decir, se planificaban grupos de 10-12 alumnos para intentar recabar fuentes primarias o ahondar en cuestiones relativas al contexto. Según la temática, había buenos "resultados heurísticos", o la visita era un paseo que ayudaba al conocimiento general, pero no aportaba a la investigación que se realizaba. Por otra parte, en algunos casos "después de los excesos sensuales de la acumulación, llega el ejercicio de sobriedad y estilización"23, ejercitación que se iba logrando tras la escritura y reescritura.

\section{Marcos teóricos-metodológicos a "medida"}

En la práctica se hizo evidente que la distancia entre la producción escrita y la idea del resultado escrito esperable no era fácil de alcanzar. Por un lado, había que aprehender "el lenguaje" y las herramientas teóricas, y por otro, había que lograr un escrito que demostrara reflexión y capacidad de elaboración pero que tuviera en cuenta, al menos, la coherencia verbal y sintáctica, como mínimo.

Josep Fontana en la presentación del libro Métodos de la Historia recomienda comenzar

22. Agradezco a Alba Ibarrola, jefa del Archivo General de la Gobernación de Misiones, la paciencia y buena predisposición que siempre brindó a cada uno de mis alumnos.

23. Lila CAIMARI: La vida en el Archivo: Goces, tedios y desvíos en el oficio de la historia, Ciudad Autónoma de Buenos Aires, Siglo XXI, 2017, p. 11. 
...a enseñar la historia como un sistema de investigación: como un conjunto de métodos cuya finalidad principal es la de ayudar a los hombres a que, a través del desciframiento de su pasado, comprendan las razones que explican su situación presente y las perspectivas que deben partir en la elaboración de su futuro. Una historia-herramienta, que enriquece la capacidad de comprensión y crítica, supone una participación activa de todos cuantos se interesan por ella ${ }^{24}$.

Esta "historia-herramienta" en la amplitud de campos de estudio históricos demandaba pensar para cada campo una metodología particular. Si bien la base para todos era la "Heurística"25, y por eso enseñar a rastrear, hallar y descubrir los vestigios, documentos y "reliquias" del tema elegido.

A los alumnos que eligieron temas de historia política, sobre las instituciones educativas, eclesiales o cooperativas/empresas yerbateras, se les recomendó la creación de fuentes orales como una metodología indispensable sobre cual partir $^{26}$.

El objeto de esta resumida exposición impide explayar las obras relativas a los estados de la cuestión particulares de cada monografía, sin embargo, se intentará sintetizar los marco-teórico-metodológicos de los diversos campos de estudio trabajados. Me hubiese gustado haber contado con material organizado como en El hilo de Ariadna ${ }^{27}$ para poder pensar desde allí los problemas metodológicos que se planteaban, no obstante, debo confesar que es un deseo "post factum".

Para las monografías sobre poblamiento y colonización, me resultaba muy útil partir del marco-teórico-metodológico realizado para mi tesis doctoral ${ }^{28}$, la cual formaba parte de los ejemplos que analizábamos. Varios alumnos me dijeron que lograron darse cuenta de lo que "yo quería" luego de leerlo; aunque no

24. Ciro Flamarión CARDOSO y Héctor PÉREZ BRIGNOLI: Los métodos de la historia: introducción a los problemas, métodos y técnicas de la historia demográfica, económica y social, Barcelona, Crítica, 1999, p. 9.

25. Enrique MORADIELLOS: El oficio..., p. 17.

26. Dora SCHWARZSTEIN: Una introducción al uso de la Historia Oral en el aula, Buenos Aires, FCE, 2001; Gabriela SCHIAVONI y Yolanda URQUIZA: Fuentes Orales, Historia y Antropología. Posadas, 2000; María Cecilia GALLERO: "Fuentes orales para el estudio de la colonización", en VV.AA: Fuentes e Interdisciplina, Actas de las II Jornadas Multidisciplinarias llevadas a cabo en Buenos Aires, 25 al 27 de agosto de 2006, Buenos Aires, CONICET- IMHICIHU, 2007, pp. 285-294; Liliana BARELA, Luis GARCÍA CONDE y Mercedes MIGUEZ: Algunos apuntes sobre historia oral y cómo abordarla, Buenos Aires, Dirección General Patrimonio e Instituto Histórico, 2012.

27. Claudia SALOMÓN TARQUINI (et. al.): El hilo de Ariadna: propuestas metodológicas para la investigación histórica, Ciudad Autónoma de Buenos Aires, Prometeo, 2019.

28. María Cecilia GALLERO: Inmigración Alemana-Brasileña en Misiones: Colonización e Identidad en la Colonia Puerto Rico, Tesis Doctoral, Mendoza, Universidad Nacional de Cuyo, 2008. 
se daban cuenta que no era un capricho mío, sino que formaba parte del oficio de observar "las leyes silenciosas que circunscriben el espacio de la operación histórica" ${ }^{\prime 29}$, en el que la combinación entre permisión e interdicción ${ }^{30}$ abría senderos y marcaba el horizonte.

Para el contexto general de las monografías de ciudades o poblados sugería empezar con el Diccionario Geográfico Toponímico de Misiones ${ }^{31}$ y la Historia de la Provincia de Misiones, Siglo XX $X^{32}$. Esta última obra también fue un puntapié para el contexto de las monografías sobre historia política o económica. Relativas a la historia reciente y la historia política se trabajaba a partir de Franco y Levin; Urquiza; Urquiza y Álvarez y Urquiza ${ }^{33}$.

Una cuestión básica que se trabajaba tras el "hallazgo de documentos" o fuentes primarias, era la clásica recomendación de la crítica externa: descubrir el origen y determinar si el documento que se analizaba era auténtico o falso; y en la crítica interna, tratar de interpretar su significado ${ }^{34}$. Más bien, ahondar la "etapa crítica" como exponen Cassani y Amuchástegui en sus cuatro momentos: "en el primero se determina si el testimonio es auténtico o fraguado; en el segundo hay que averiguar el grado de verdad que encierra la noticia contenida en él; el tercero consiste en interpretar el testimonio según las distintas implicaciones previsibles; el último, en establecer su valor con relación al tema investigado y a posibles derivaciones" ${ }^{\prime 35}$.

En relación a la metodología, una base importante fue la obra de Peter Burke, Formas de hacer historia. Los capítulos más utilizados fueron "Sobre microhistoria" e "Historia Oral"36. Con respecto a la primera, también servía de ejemplo el libro de Jacques Revel, Un momento historiográfico ${ }^{37}$, pues la vía

29. Michel DE CERTEAU: "La operación...", p. 16.

30. Ibid. p. 31.

31. Miguel Ángel STEFAÑUK: Diccionario Geográfico Toponímico de Misiones, Buenos Aires, Contratiempo, 2009.

32. María Angélica AMABLE, Karina DOHMANN y Liliana Mirta ROJAS: Historia de la Provincia de Misiones, Siglo XX, Posadas, Ediciones Montoya, 2008.

33. Marina FRANCO y Florencia LEVÍN (comp.): Historia reciente. Perspectivas y desafíos para un campo en construcción, Buenos Aires, Paidós, 2007; Yolanda URQUIZA: Las prácticas políticas en los partidos radical y peronista: 1947-1997, Tesis doctoral, Barcelona, Universidad de Barcelona, Mimeo, 2005; Yolanda URQUIZA y Norma ÁLVAREZ: Misiones. Entre la provincialización y la dictadura, Posadas, UNaM, 2010; y Yolanda URQUIZA: Misiones bajo el terror 1976-1983, Misiones, s.e, 2010.

34. Jacques LE GOFF (dir.): Pensar..., p. 108.

35. Jorge CASSANI y A. J. AMUCHÁSTEGUI: Del Epos a la historia científica, Buenos Aires, Nova, 1961, p. 219.

36. Peter BURKE (ed.): Formas de hacer historia, Madrid, Alianza, 1993; Giovanni LEVI: "Sobre Microhistoria", en Peter BURKE (ed.): Formas..., pp. 119-143; y Gwyn PRINS: "Historia Oral", en Peter BURKE (ed.): Formas..., pp. 144-176.

37. Jacques REVEL: Un momento historiográfico: Trece ensayos de historia social, Buenos Aires, Manantial, 2005, p. 46. 
microhistórica es profundamente diferente en tanto en sus intenciones como en sus procedimientos. "Cambiar el foco del objetivo no es solamente aumentar (o disminuir) el tamaño del objeto en el visor, sino también modificar la forma y la trama"38. En palabras de Giovanni Levi, "la microhistoria es por esencia una práctica historiográfica", su método se interesa ante todo y sobre todo por los procedimientos concretos y detallados que constituyen la obra del historiador, y en cuanto práctica, "se basa en esencia en la reducción de la escala de observación, en una análisis microscópico y en un estudio intensivo del material documental" 39 .

De modo que en mi enseñanza el hincapié estuvo dado en el estudio intensivo de los materiales documentales obtenidos y en alternar con enfoques generales que permitieran contextualizar los procesos a escala provincial o regional, aspecto en el cual era fundamental el conocimiento de bibliografía general e historiográfica.

En las historias de empresas el análisis microhistórico era la opción preferida, pues la falta de conocimientos en economía impedía realizar estudios cuantitativos o análisis sobre precios, ventas, etc. Por lo general se partía de la obra de María Inés Barbero ${ }^{40}$, y luego se optaba por hacer un análisis de los orígenes de la empresa, algunos vaivenes de crecimiento o retracción, para lo cual era útil contextualizar el tema en base a "La economía de Misiones" ${ }^{41}$.

Introducir nuevas corrientes historiográficas fue un desafío aceptado por pocos, algunos incursionaron en historia de género ${ }^{42}$ y la familia ${ }^{43}$; otros pocos, en historia ambiental, en la cual la apoyatura teórica estaba basada en Gallini y Bugallo o en fundamentos de la propia geografía histórica ${ }^{44}$.

38. Jacques REVEL: Un momento..., p. 46.

39. Giovanni LEVI: "Sobre Microhistoria...", p. 122.

40. María Ines BARBERO: Historia de empresas aproximaciones historiográficas y problemas en debate, Buenos Aires, Centro Editor de América Latina, 1993.

41. Miguel Ángel FREAZA: Economía de Misiones: aspectos y actividades relevantes, período 1980-1999. Posadas, UNaM, 2002.

42. Cecilia LAGUNAS: "Historia y género. Algunas consideraciones sobre la historiografía feminista", La aljaba, segunda época, Vol. I (1996), pp. 27-33; y Dora Beatriz BARRANCOS: "Estudios de género y renovación de las Ciencias Sociales en Argentina", Revista Horizontes Sociológicos, 1 (enero-junio 2013), pp. 224-237.

43. Mónica BERJ y Roxana BOIXADÓS: La Familia. Campo de investigación interdisciplinario, Buenos Aires, Universidad Nacional de Quilmes, 2004.

44. Stefania GALLINI: "Problemas de Métodos en la Historia Ambiental de América Latina", Anuario IHES (Argentina), 19 (2004), pp. 147-171; y Stefania GALLINI: "Invitación a la historia ambiental", Revista Tareas, 120 (mayo-agosto 2005), pp. 5-28; Irene BUGALLO: Filosofía Ambiental y Ecosofías: Arne Naess, Spinoza y James, Buenos Aires, Prometeo, 2015; Carl SAUER, "Introducción a la geografía histórica". Polis, Revista de la Universidad Bolivariana, vol. 3, 8 (2004), s.n. 
Para las historias de instituciones escolares se intentaba aplicar la microhistoria, aunque por lo general se quedaban en una descripción de la escuela y el contexto que la vio surgir, siendo útiles para este marco los capítulos de Teresa Artieda sobre la Historia de la Educación en Misiones ${ }^{45}$. Admito que el objetivo de lograr analizar "la historia de la educación" o los cambios en el "acto educativo" quedaron truncos. Quizás porque el horizonte era muy lejano y la meta de reconstruir la historia de la escuela en sí era más viable. Un problema similar se planteaba con los trabajos de Historia Cultural, pues al igual que plantea Peter Burke en su introducción "una solución al problema de definir la historia cultural podría pasar por desplazar la atención de los objetos a los métodos de estudio", lo cual plantea controversias, pues unos cuantos intentan emplear métodos cuantitativos. Algunos describen su trabajo como una búsqueda de significados, otros se centran en las prácticas y las representaciones. Unos conciben su objetivo como esencialmente descriptivo, otros creen que la historia cultural, como la historia política, puede y debería presentarse como un relato ${ }^{46}$.

El tema es que hay diferentes relatos, o bien, diferentes modos de encarar ese relato, y muchas veces la "reconstrucción" de la trama histórica se va hilvanando en una sucesión de hechos. Usando una metáfora de costureras, podría decirse que el nivel de análisis pasaría de simples pespuntes a una costura diseñada y bien lograda. Sin embargo, para lograr esa costura final, hay que saber cortar bien el molde, usar bien la trama y tener en cuenta la textura de la tela. Aunque me hubiese gustado tener moldes prediseñados para darle a cada alumno, fue necesario ir construyendo y recortando el mismo a medida que avanzaba la investigación y las lecturas teóricas.

Hacia el final se trabajaba a partir del Antimanual del mal historiador ${ }^{47}$, sobre "los siete (y más) pecados capitales del mal historiador", en los que se van sucediendo el positivismo, "que degrada a la ciencia de la historia a la simple y limitada actividad de la erudición"; el anacronismo; la noción del tiempo; la idea limitada del progreso; una actitud acrítica; el mito repetido de su búsqueda de una "objetividad" y "neutralidad" absoluta frente a su objeto de estudio, y por último, el "pecado del posmodernismo" que desconoce la historia real y aboga por los discursos históricos ${ }^{48}$.

45. Teresa ARTIEDA: "El Magisterio en los Territorios Nacionales: el caso de Misiones" y "Temas en el escenario educativo de Misiones (1946-1985)", ambos capítulos en Adriana PUIGGRÓS (dir.): La educación en las Provincias y Territorios Nacionales (1885-1945), Buenos Aires, Editorial Galerna, 1993, pp. 167-209 y pp. 300-342 respectivamente.

46. Peter BURKE: ¿Qué es la historia cultural?, Barcelona, Espasa Libros, 2006, p. 15

47. Carlos Antonio AGUIRRE ROJAS: Antimanual del mal historiador, México, La vasija \& Prohistoria, 2002.

48. Carlos Antonio AGUIRRE ROJAS: Antimanual..., pp. 30-45, cursivas y comillas en el original. 
Asimismo, era importante recalcar y ejemplificar "cuándo y cómo se cita: diez reglas" de la obra Cómo hacer una tesis de Umberto $\mathrm{ECO}^{49}$.

Finalmente se daban algunas pautas para la exposición oral llegado el momento de la defensa del trabajo en el examen final.

\section{Conclusiones sobre la práctica docente en la investigación histórica}

Resumir la experiencia de mi práctica docente en la investigación histórica fue quizás un modo de hacer catarsis, quizás también un modo de tomar distancia sobre mi propio accionar. Una cosa es analizar los resultados grupales, otra muy diferente el proceso individual de cada alumno: en su interés por investigar fueron ampliando y retroalimentando mis conocimientos sobre la historia misionera y regional.

La tensión entre lo que enseñaba, lo que ellos lograban escribir y las correcciones que realizaba eran tironeadas por sentimientos ambivalentes. La escritura de la monografía resultaba un trabajo arduo. Los estudiantes debían reflejar su pensamiento y los descubrimientos de los documentos -información- sucesos y analizarlos dejando en evidencia la metodología adoptada. En muchos casos, realizar largas citas - haciendo uso del collage de "cortar y pegar"- era una tentación inevitable, difícil de corregir. Sin embargo, lograr que fueran desarrollando su propio pensamiento, dando coherencia a los descubrimientos, organizando los capítulos, daba satisfacciones que son difíciles de resumir en un artículo.

La elaboración de una monografía implicaba una serie procedimientos textuales y cognitivos bastante complejos, en los cuales las falencias o fortalezas adquiridas durante la educación secundaria eran evidentes ${ }^{50}$. Aunque se estaba llegando al final del profesorado, las faltas en una preparación adecuada en aspectos fundamentales de lectura y escritura continuaban arrastrándose en algo tan necesario como era el proceso de escritura reflexiva. En este sentido, las exigencias que implica redactar una monografía ponían en juego un conjunto de interacciones y saberes que estaban interconectados. Fue interesante mirar en retrospectiva el tiempo que llevó a cada alumno llegar a la instancia del examen final, en general un $67 \%$ rindieron en el mismo año o en el transcurso del siguiente. Lo cual hizo evidente que la disposición para trabajar en las horas de clase, aprovechar las visitas a la biblioteca y el archivo era tiempo ganado si era bien utilizado. En el examen

49. Umberto ECO: Cómo hacer una tesis, Barcelona, Gedisa, 1998, pp. 188-197.

50. En este punto es interesante considerar la "construcción del conocimiento profesional docente", que plantean Irma Rosso y Teresa Alcalá en "La formación docente y el ejercicio profesional de los noveles profesores en historia". Revista del Instituto de Investigaciones en Educación Facultad de Humanidades-UNNE, 7, 9 (2016), pp. 49-70. 
final, por otra parte, muchos alumnos lograron dar un oral en el que el trabajo se lucía y dejaban en claro sus descubrimientos, en tanto que para otros, no era tan fácil iluminar las vicisitudes que habían atravesado hasta llegar a ese momento.

Una dificultad generalizada para los marcos teóricos fue que los diccionarios históricos se refieren a conceptos o procesos europeos, relativos a hechos o sucesos imposibles de encajar en la realidad misionera ${ }^{51}$. Los mismos servían para tomar como referencia, con la consiguiente salvedad y explicación de su aplicación en la historia regional. Quizás era un modo de lentamente ir produciendo un "ensanchamiento del horizonte histórico que debe llevar a una conmoción de la ciencia histórica. Es la necesidad de poner fin al etnocentrismo, la necesidad de deseuropeizar la historia" ${ }^{\prime \prime 2}$.

Otra dificultad estuvo dada en que los libros de metodología quedaban lejanos en su real aplicabilidad ${ }^{53}$, o que parecían manuales de historia social pero eran sobre historia argentina direccionada para la Licenciatura de Trabajo Social ${ }^{54}$. El manual de Topolski sobre Metodología de la Historia ${ }^{55}$, aunque recomendado y disponible en la biblioteca, fue utilizado excepcionalmente. Más aceptados fueron los manuales sobre cómo hacer una tesis ${ }^{56}$.

La escritura de la Historia en espacios locales y regionales evidencia complejidades atravesadas por "permisiones" e "interdicciones", intereses personales, obligación de pasar un examen, en el cual, el ánimo de la cátedra era enseñar un "oficio" y dar cuenta que: "Jamás tenemos convicciones absolutas cuando se trata de hechos históricos... El historiador no es el que sabe. Es el que investiga" ${ }^{\prime \prime}$.

51. Pierre VILAR: Iniciación al vocabulario del análisis histórico, Barcelona, Crítica, 1999; Elena SÁNCHEZ de MADARIAGA: Conceptos fundamentales de Historia, Madrid, Alianza, 2009; André BURGUIÈRE (dir.): Diccionario Akal de Ciencias Históricas, Madrid, Akal, 2005.

52. Jacques LE GOFF (dir.): Pensar..., p. 134.

53. Néstor COHEN y Juan Ignacio PIOVANI: La metodología de la investigación en debate, Buenos Aires, Eudeba, 2008; Ruth SAUTU (et. al.): Manual de metodología: construcción del marco teórico, formulación de objetivos y lección de la metodología, Buenos Aires, PrometeoClacso, 2010; Aldo MERLINO (coord.): Investigación cualitativa en Ciencias Sociales, Buenos Aires, Cengage Learning Argentina, 2009; y Roberto FERNÁNDEZ SAMPIERI (et. al.): Manual de Metodología, México, Mc Graw Hill, 2014.

54. Mariela CEVA, Aníbal JÁUREGUI y Julio STORNINI, Manual de Historia Social Argentina. Buenos Aires, Prometeo, 2010.

55. Jerzy TOPOLSKI: Metodología de la historia, Madrid, Cátedra. Historia, 1985.

56. Huáscar TABORGA: Cómo hacer una tesis, México, Grijalbo, 1997; Hugo MANCUSO: Metodología de la investigación en ciencias sociales, Buenos Aires, Paidós, 1999; María Cecilia SOUZA MINAYO (org.): Investigación Social. Teoría, método y creatividad, Buenos Aires, Lugar Editorial, 2003; y Mirta BOTTA: Tesis, tesinas, monografías e informes, Buenos Aires, Biblos, 2007.

57. Paul CHALUS: "Presentación", en Lucien FEBVRE: El problema de la incredulidad en el siglo XVI. La religión de Rabelais, Madrid, Akal, 1993, pp. 5-6. 


\section{Bibliografía}

AGUIRRE ROJAS, Carlos Antonio: Antimanual del mal historiador, México, La vasija \& Prohistoria, 2002.

AMABLE María Angélica: La documentación histórica en los Archivos Provinciales, Posadas, Tesis de Grado Inédita, Universidad Nacional de Misiones, Facultad de Humanidades y Ciencias Sociales, Noviembre, 1996.

AMABLE, María Angélica, DOHMANN, Karina y ROJAS, Liliana Mirta: Historia de la Provincia de Misiones, Siglo XX, Posadas, Montoya, 2008.

ARENDT, Hannah: De la historia a la acción, Barcelona, Paidós, [1ª ed. 1953] 1998.

ARÓSTEGUI, Julio: La investigación Histórica: Teoría y Método, Barcelona, Crítica, 2001.

ARTIEDA, Teresa: "El Magisterio en los Territorios Nacionales: el caso de Misiones", en Adriana PUIGGRÓS (dir.): La educación en las Provincias y Territorios Nacionales (1885-1945), Buenos Aires, Galerna, 1993, pp. 167-209.

ARTIEDA, Teresa: "Temas en el escenario educativo de Misiones (1946-1985)", en Adriana PUIGGRÓS (dir.): La educación en las Provincias y Territorios Nacionales (1885-1945), Buenos Aires, Galerna, 1993, pp. 300-342.

ASTRAIN, Antonio: Jesuitas, Guaraníes y Encomenderos: historia de la Compañía de Jesús en el Paraguay, Asunción, CEPAG, 1995.

BARBERO, María Inés: Historia de empresas aproximaciones historiográficas y problemas en debate, Buenos Aires, Centro Editor de América Latina, 1993.

BARELA, Liliana, GARCÍA CONDE, Luis y MIGUEZ, Mercedes: Algunos apuntes sobre historia oral y cómo abordarla, Buenos Aires, Dirección General Patrimonio e Instituto Histórico, 2012.

BARRANCOS, Dora Beatriz: "Estudios de género y renovación de las Ciencias Sociales en Argentina", Revista Horizontes Sociológicos, 1 (enerojunio 2013), pp. 224-237.

BERJ, Mónica y BOIXADÓS, Roxana: La Familia. Campo de investigación interdisciplinario, Buenos Aires, Universidad Nacional de Quilmes, 2004.

BLOCH, Marc: Introducción a la Historia, México, FCE, 2000.

BOTTA, Mirta: Tesis, tesinas, monografías e informes, Buenos Aires, Biblos, 2007.

BRAGONI, Beatriz: Microanálisis, Buenos Aires, Prometeo, 2004.

BRAUDEL, Fernand: La Historia y las Ciencias Sociales, Madrid, Alianza, 1970.

BRIE, Roberto: Los hábitos del pensamiento riguroso, Buenos Aires, Ed. del Viejo Aljibe, 1998.

BUCHBINDER, Pablo: “¿Qué debe saber un historiador? Reflexiones sobre los modelos curriculares y la enseñanza superior de la historia en la Argentina durante el siglo XX", Clio \& Asociados 15 (2011), pp. 157-177. 
BUGALLO, Irene: Filosofía Ambiental y Ecosofías: Arne Naess, Spinoza y James, Buenos Aires, Prometeo, 2015.

BURGUIÈRE, André (dir.): Diccionario Akal de Ciencias Históricas, Madrid, Akal, 2005.

BURKE, Peter (ed.): Formas de hacer historia, Madrid, Alianza, 1993.

BURKE, Peter: ¿Qué es la historia cultural?, Barcelona, Espasa Libros, 2006.

CAIMARI, Lila: La vida en el Archivo: Goces, tedios y desvíos en el oficio de la historia, Ciudad Autónoma de Buenos Aires, Siglo XXI, 2017.

CARDOSO, Ciro Flamarión: Introducción al trabajo de la investigación histórica, Barcelona, Crítica, 1989.

CARDOSO, Ciro Flamarión y PÉREZ BRIGNOLI, Héctor: Los métodos de la historia: introducción a los problemas, métodos y técnicas de la historia demográfica, económica y social, Barcelona, Crítica, 1999.

CASSANI, Jorge y PÉREZ AMUCHÁSTEGUI, J. A.: Del Epos a la historia científica, Buenos Aires, Nova, 1961.

CEVA, Mariela, JÁUREGUI, Aníbal y STORNINI, Julio: Manual de Historia Social Argentina, Buenos Aires, Prometeo, 2010.

CHALUS, Paul: "Presentación", en Lucien FEBVRE: El problema de la incredulidad en el siglo XVI. La religión de Rabelais, Madrid, Akal, 1993.

COHEN, Néstor y PIOVANI, Juan Ignacio: La metodología de la investigación en debate, Buenos Aires, Eudeba, 2008.

DE CERTEAU, Michel: "La operación histórica", en Jacques LE GOFF (ed.): Hacer la historia, Barcelona, Laia, 1984.

ECO, Umberto: Cómo hacer una tesis, Barcelona, Gedisa, 1998.

FERNANDEZ SAMPIERI, Roberto (et. al.): Manual de Metodología, México, McGraw Hill, 2014.

FRANCO, Marina y LEVÍN, Florencia (comp.): Historia reciente. Perspectivas y desafíos para un campo en construcción, Buenos Aires, Paidós, 2007.

FREAZA, Miguel Ángel: Economía de Misiones: aspectos y actividades relevantes, período 1980-1999. Posadas, UNaM, 2002.

FURLONG, Guillermo: Misiones y sus Pueblos Guaraníes, Buenos Aires, Imprenta Balmes, 1962.

GALLERO, María Cecilia: "Fuentes orales para el estudio de la colonización", en Fuentes e Interdisciplina, Actas de las II Jornadas Multidisciplinarias Ilevadas a cabo en Buenos Aires, 25 al 27 de agosto de 2006, Buenos Aires, CONICET- IMHICIHU, 2007, pp. 285-294.

GALLERO, María Cecilia: Inmigración Alemana-Brasileña en Misiones: Colonización e Identidad en la Colonia Puerto Rico, Tesis Doctoral, Mendoza, Universidad Nacional de Cuyo, 2008. 
GALLINI, Stefania: "Problemas de Métodos en la Historia Ambiental de América Latina", Anuario IHES (Argentina), 19 (2004), pp. 147-171.

GALLINI, Stefania: "Invitación a la historia ambiental", Revista Tareas, 120 (mayo-agosto 2005), pp. 5-28.

GAMALLO, Gustavo: "La "publificación" de las escuelas privadas en Argentina", Revista SAAP. Publicación de Ciencia Política de la Sociedad Argentina de Análisis Político vol. 9, 1 (2015), pp. 43-74.

GINZBURG, Carlo: El hilo y las huellas. Lo verdadero, lo falso, lo ficticio, Buenos Aires, FCE, 2010.

GOBIERNO DE MISIONES, Gran Atlas de Misiones. Posadas, IPEC, 2012.

GUBER, Rosana: La etnografía, método, campo y reflexividad, Buenos Aires, Siglo XXI, 2012.

JAQUET, Héctor: Haciendo Historia en la Aldea, Misiones, 1996, Misiones, s.e, 2002.

JAUME, Fernando, GONZALEZ VILLAR, Carlos, URQUIZA, Yolanda y SINTES, Lila: Notas sobre la Historia de Misiones, El proceso de Constitución de la Región Histórica, Documentos de Trabajo, Proyecto POBUR, UNaM-CONICET, [s.f.].

LAGUNAS, Cecilia: "Historia y género. Algunas consideraciones sobre la historiografía feminista", La aljaba, segunda época, Vol. I (1996), pp. 27-33.

LE GOFF, Jacques (dir.): Pensar la Historia, Barcelona, Paidós, 2005.

LESCANO, María Eva y VENANZI, Silvia: 1960-2010, Instituto Superior Antonio Ruiz de Montoya, 50 Aniversario, Posadas, Montoya, 2010.

LEVI, Giovanni: "Sobre Microhistoria", en Peter BURKE (ed.): Formas de hacer historia, Madrid, Alianza, 1993, pp. 119-143.

MAEDER, Ernesto: "Los Archivos del Nordeste", Boletín Interamericano de Archivos, Volumen VIII (1981), pp. 177-183.

MAEDER, Ernesto: Aproximación a las misiones guaraníticas, Buenos Aires, EDUCA, 1996.

MANCUSO, Hugo: Metodología de la investigación en ciencias sociales, Buenos Aires, Paidós, 1999.

MARROU, Henri: Del Conocimiento Histórico, Buenos Aires, Per Abbat, 1985.

MERLINO, Aldo (coord.): Investigación cualitativa en Ciencias Sociales, Buenos Aires, Cengage Learning Argentina, 2009.

MORADIELLOS, Enrique: El oficio de historiador, Madrid, Siglo XXI, 2005.

PARDINAS, Felipe: Metodologías y técnicas de Investigación en Ciencias Sociales, México, Siglo XXI, 1979.

PERIÉ DE SCHIAVONI, Ángela: "Reflexiones metodológicas sobre la construcción de la historia regional", Revista de Estudios Regionales, año 11, 22 (2002), pp. 7-11. 
PRINS, Gwyn: "Historia Oral", en Peter BURKE (ed.): Formas de hacer historia, Madrid, Alianza, 1993, pp. 144-176.

REVEL, Jacques: Un momento historiográfico: Trece ensayos de historia social, Buenos Aires, Manantial, 2005.

ROSSO, Irma y ALCALÁ, Teresa: "La formación docente y el ejercicio profesional de los noveles profesores en historia", Revista del Instituto de Investigaciones en Educación Facultad de Humanidades-UNNE. 7, 9 (2016), pp. 49-70.

SÁNCHEZ de MADARIAGA, Elena: Conceptos fundamentales de Historia, Madrid, Alianza, 2009.

SAUER, Carl: "Introducción a la geografía histórica", Polis, Revista de la Universidad Bolivariana, vol. 3, 8 (2004) https://www.redalyc.org/articulo. oa?id=30500821 [Consultado 7/1/2017]

SAUTU, Ruth (et. al.): Manual de metodología: construcción del marco teórico, formulación de objetivos y lección de la metodología, Buenos Aires, Prometeo-Clacso, 2010.

SCHIAVONI, Gabriela y URQUIZA, Yolanda: Fuentes Orales, Historia y Antropología, Posadas, UnaM, 2000.

SCHWARZSTEIN, Dora: Una introducción al uso de la Historia Oral en el aula, Buenos Aires, FCE, 2001.

SOUZA MINAYO, María Cecilia (org.): Investigación Social. Teoría, método y creatividad, Buenos Aires, Lugar Editorial, 2003.

STEFAÑUK, Miguel Ángel: Diccionario Geográfico Toponímico de Misiones, Buenos Aires, Contratiempo, 2009.

TABORGA, Huáscar: Cómo hacer una tesis, México, Grijalbo, 1997.

SALOMÓN TARQUINI, Claudia (et. al.): El hilo de Ariadna: propuestas metodológicas para la investigación histórica, Ciudad Autónoma de Buenos Aires, Prometeo, 2019.

TOPOLSKI, Jerzy: Metodología de la historia, Madrid, Cátedra. Historia, 1985.

URQUIZA, Yolanda: "Tras las Huellas de la Ciudadanía en un Territorio de Frontera", Cuadernos del Sur-Serie Historia, 30-31 (2002), pp. 155-185.

URQUIZA, Yolanda: Las prácticas políticas en los partidos radical y peronista: 1947-1997, Tesis doctoral, Barcelona, Universidad de Barcelona, Mimeo, 2005.

URQUIZA, Yolanda: Misiones bajo el terror 1976-1983, Misiones, s.e., 2010.

URQUIZA, Yolanda y ÁLVAREZ, Norma: Misiones. Entre la provincialización y la dictadura, Posadas, UNaM, 2010.

VILAR, Pierre: Iniciación al vocabulario del análisis histórico, Barcelona, Crítica, 1999. 